\title{
Adding Sputum Collection and Transportation Services for Early Identification TB Cases in Hard-to-Reach Difficult Terrain-Will It Help?
}

\author{
Badri Thapa, Banuru Muralidhara Prasad*, Sarabjit Singh Chadha, Subrat Mohanty, \\ Deepak R. Mishra, Jamie Tonsing
}

TB and Communicable Disease Department, International Union against Tuberculosis and Lung Disease, South-East Asia Regional Office, New Delhi, India

Email: ^drprasadbm@gmail.com

How to cite this paper: Thapa, B., Prasad, B.M., Chadha, S.S., Mohanty, S., Mishra, D.R. and Tonsing, J. (2017) Adding Sputum Collection and Transportation Services for Early Identification TB Cases in Hard-toReach Difficult Terrain-Will It Help? Journal of Tuberculosis Research, 5, 220-228. https://doi.org/10.4236/jtr.2017.54023

Received: August 30, 2017

Accepted: October 30, 2017

Published: November 2, 2017

Copyright (c) 2017 by authors and Scientific Research Publishing Inc. This work is licensed under the Creative Commons Attribution International License (CC BY 4.0).

http://creativecommons.org/licenses/by/4.0/

CC) $\underset{\mathrm{EY}}{\mathrm{EY}}$ Open Access

\begin{abstract}
A community engagement project was designed and implemented to reach 10.6 million population in 13 districts of Uttarakhand state-India, to identify presumptive TB patients (PTBPs) and link to free diagnostics services, either through referral or sputum collection and transportation (SCT). The objective of this study is to determine additional yield of TB patients achieved by providing SCT services in comparison to referrals of PTBPs identified in hard-to-reach areas. This was before and after comparative study conducted between April-June 2014 (2Q2014-before) to July-September 2014 (3Q2014-after). SCT was advised for all PTBPs identified from community level interventions between July-September 2014 (3Q2014-after). In 3Q2014, additional 279 (34\%) PTBPs were examined in the microscopy centre with an additional 36 (51\%) new smear positive (NSP) patient diagnosed. A total of 46 NSP (128\%) were contributed by SCT alone. Higher proportion (94\%) of TB patients was initiated on treatment in 3Q2014 in comparison to 2Q2014 (91\%). SCT as a strategy for early case detection in hard-to-reach difficult terrain yields positive results in comparison to mere referral of PTBPs.
\end{abstract}

\section{Keywords}

Hard-to-Reach, Sputum Collection and Transportation, India, Tuberculosis, Project Axshya

\section{Introduction}

The recent Global TB report raised concerns over the missing "million" Tuberculosis (TB) cases and this is believed to be concentrated around countries with 
high TB burden-including India [1]. The burden is believed to be under-reported as a million cases (estimated) are being missed from routine programme reporting system [2]. Few evidences relate missing cases to: lack of awareness in community about TB services provided by government, prevailing stigma and misconceptions about TB, diagnosis and treatment seeking behaviour, low notification from heterogeneous private sector who are first point of contact, and missed diagnosis after the patients reaches the microscopy centers under programme [3] [4] [5] [6].

Taking cognisance of the Global Technical Strategy; Government of India, developed National Strategic Plan for TB (2012-17) with an aim to reach, sensitize and engage, communities in hard-to-reach areas for TB prevention and care, thereby reduce the missing million cases [7] [8]. In lieu with global and national strategy, The International Union against Tuberculosis and Lung Disease (The Union), South East Asia Office launched Project Axshya (meaning TB free) to support the programme with an objective to engage civil society to increase awareness about TB in community through Advocacy, Communication, and Social Mobilization (ACSM) strategy. The project, Project Axshya, was implemented in 300 districts of India across 21 states and reaching millions of households with support from The Global Fund [9].

Project Axshya implemented series of community engagement activities through a network of Non-governmental Organizations (NGOs) and community volunteers. Local community volunteers were identified and trained, who would then visit the mapped villages, interact with village members and inform them about Tuberculosis, prevention and care. All Presumptive TB Patients (PTBPs) identified during the community level activities would then be referred for diagnosis at publicly funded microscopy centers (MC). In addition sputum collection and transportation (SCT) services were also provided for needy PTBPs. Over the period, identified PTBPs were given the choice of referral or SCT services through volunteers and the additional yield of TB patients through SCT services over referrals were not known. Therefore, the objective of this study is to determine additional yield of TB patients achieved by providing SCT services in comparison to referrals of PTBPs identified in hard-to-reach areas.

\section{Materials and Methods}

\subsection{Study Setting, Design and Interventions}

The study was conducted between April-June 2015 in 13 districts of Uttarakhand state-India, with the before (2Q2014, April-June 2014) and after (3Q2014, JulySeptember 2014) study design. Uttarakhand is located in the northern India, with sparsely disbursed population (10.6 million) and $70 \%$ of population lives in rural area, $69 \%$ of them are literate, $39 \%$ are daily wage earners and agriculture is the main economy. The state is $86 \%$ mountainous and $15,436 \mathrm{~km}$ of rural roads with infrequent, expensive and limited transportation services [10]. From the recently published programme report, the annual smear positive TB case notifi- 
cation rate for the state was at 125 and annual total TB case notification rate was at 136 per 100,000 populations [2].

Project Axshya, an ACSM project was implemented through identified and trained local NGOs and CVs in the mapped villages of the districts in Uttarakhand. The community activities were monitored and supervised by district level project staff-District Coordinator (DC). The activities included, community meetings at village level (awareness among women groups, youth groups, school children, village health sanitation group etc., and identified PTBPs (if any) through these groups), and mid-media activities (creating TB awareness through wall painting, public announcements using mike speakers, street plays etc.) [11]. In addition, CVs made house-to-house visits (active case finding-ACF), to create awareness about TB which is known as Axshya SAMVAD (sensitization, awareness among marginalized and vulnerable areas of district). Local healthcare providers in the village-Rural Healthcare providers (RHCPs) were sensitized and engaged in the project who were regularly visited by CVs for referrals or SCT of PTBPs to microscopy centers (MC) (see Figure 1). This holistic approach led to identification of PTBPs who were referred for examination and those who were found sputum positive were put on treatment as per programme guideline [12].

Community volunteers engaged under the NGOs were given two day training on TB basics-signs and symptoms, diagnosis, treatment, referrals and sputum collection and transportation. The main criteria for identification of PTBPs during the activities (outlined above) or during the visit to house-to-house were 1) cough $\geq 2$ weeks 2 ) weight loss 3 ) loss of appetite 4) evening rise in temperature.

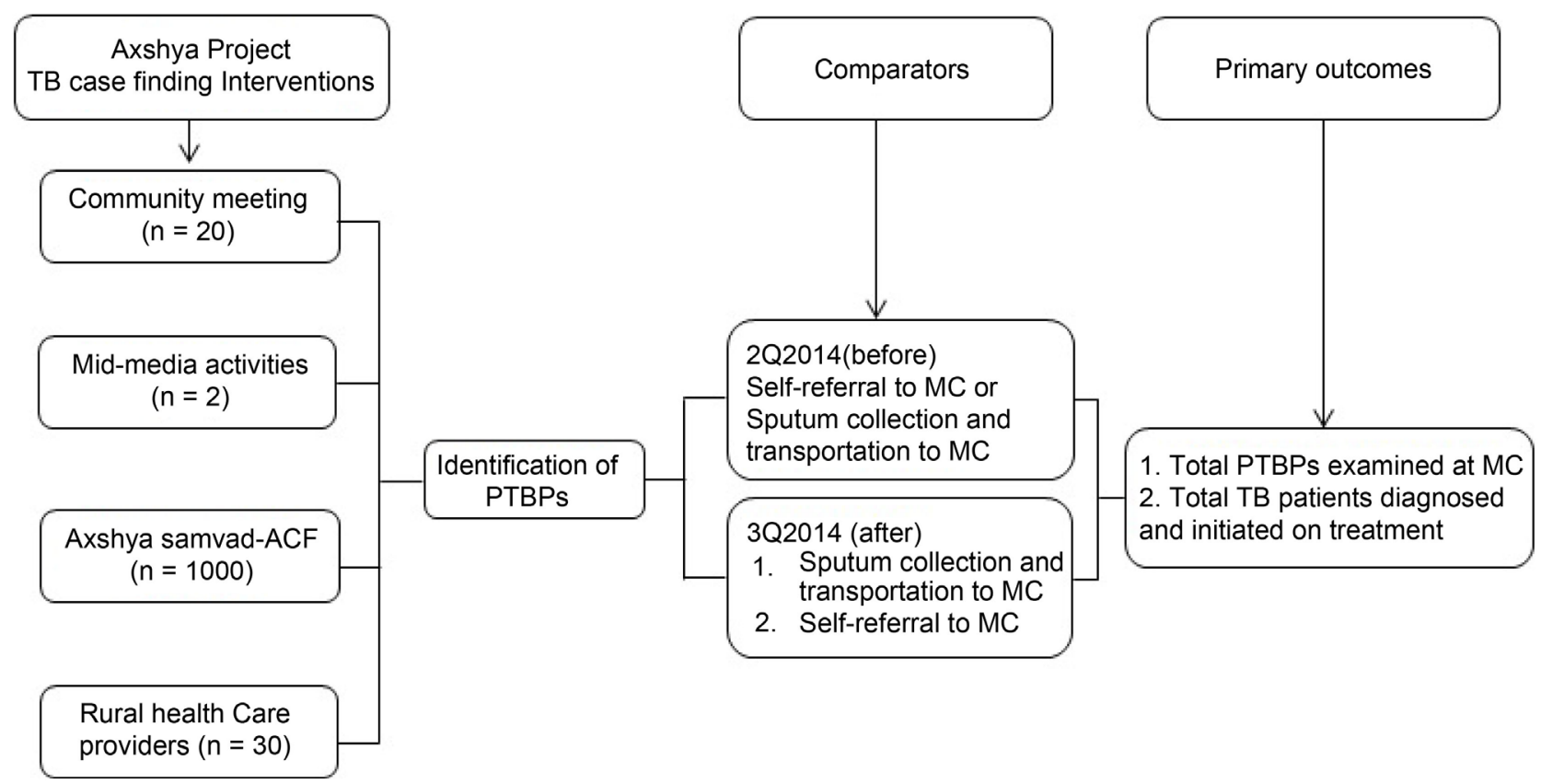

Figure 1. Study design flow chart. Abbreviations: n-number per month per district; per ACF-active case finding; PTBPspresumptive TB patients; MC—microscopy centre; TB_tuberculosis; 2Q2014-April-June 2014; 3Q2014-July-September 2014. 
Those who had these symptoms were given the choice of referral or SCT services. For SCT services, CVs were trained to collect quality sputum and adhere to infection control measures while transporting the sputum. CVs were paid sum of Rs 100 (USD 1.62) for two samples collected per PTBPs identified. Payment was made when the sputum smear positivity was at $7 \%$ of the total samples collected. This cut-off was arrived using programme and project data.

Of the total 10.6 million, about 400,000 individuals were reached through the project activities during the 6 months and were included in the study for screening of PTBPs, diagnosis and treatment. In 2Q2014, the project referred identified PTBPs residing within $5 \mathrm{~km}$ (in hilly area) and $10 \mathrm{~km}$ (plain area) of nearest public health facility with microscopy centre (MC) for sputum smear examination. PTBPs who did not meet these criteria were given a choice of referral or SCT (two samples-spot and morning samples). Only $18 \%$ of those referred reached the MC. In 3Q2014, the strategy was revised to offer SCT services for all PTBPs irrespective of the distance from the MC. PTBPs denying the SCT services were referred with a referral slip. The results were collected by the CVs and communicated to PTBPs and those smear positives were initiated on treatment as per National Tuberculosis Programme (NTP) guideline [12]. The project referrals and SCTs were recorded in the special forms designed for the project and TB laboratory register of the MC. The project staff at district level randomly verified about $10 \%$ of all PTBTs and validated the receipt of services during the routine monitoring and supervision visits. Second level of monitoring was conducted by programme managers placed at the state head quarter.

\subsection{Data Collection, Entry, and Analysis}

The aggregated secondary data collected from project forms of community volunteers included variables-number of household visited, community meetings and mid-media activity conducted, PTBPs identified, total referred and SCT done for smear examination. In addition to these variables we analysed data collected by volunteers regarding reasons for referrals not reaching MC during their follow-up visits. The consolidated number of PTBPs identified, examined, total smear positive TB patients diagnosed and initiated on treatment were analysed. The comparison of proportions was done by Chi-square test. The p-value $\leq$ 0.05 were considered statistically significant. This study did not use individual patient information and identifiers. The Ethics Advisory Group of the International Union Against Tuberculosis and Lung Disease (The Union), Paris, France approved the study (approval no 17/16).

\section{Results}

In 2Q2014, 30,835 households were covered through active case finding activity (ACF-known as Axshya SAMVAD), organized 641 community meetings and sensitized 138 RHCPs while in 3Q2014, 45,068 households were visited through ACF (48\% increase), organized 744 community meetings (16\% increase) and 
sensitized 135 RHCPs (2\% decrease).

In 2Q2014, 200,000 individuals were reached through these activities and 1907 PTBPs were identified. Of these, 1413 (73\%) were referred and 261 (18\%) were examined. Of 1152 loss to referrals (LTR), reasons were obtained from 975 PTBPs. Most common reasons were; MC not accessible (19\%), no adequate follow up of referrals (17\%), loss of daily wages (15\%) and pre-occupied with agricultural activities (12\%) (Table 1). Almost $73 \%$ of the LTR could have been examined by promoting SCT.

In 3Q2014, 200,000 individuals were reached, of which 2321 PTBPs were identified and 241 reached the MC. The referrals declined from 74\% in 2Q2014 to $63 \%$ in $3 \mathrm{Q} 2014$ ( $\mathrm{p} \leq 0.001$ ) with no significant difference in referrals reaching the MC (18\%-2Q2014 vs 16\%-3Q2014) ( $\mathrm{p}=0.14)$ (Table 2). SCT services was promoted in 3Q2014, which almost doubled from 494 in 2Q2014 to 855 in 3Q2014 with an additional 361 SCTs $(73 \%)$ ( $\mathrm{p} \leq 0.001)$. SCT led to more PTBPs examination in 3Q2014 $(\mathrm{n}=1096,47 \%)$ in comparison to 2Q2014 ( $\mathrm{n}=817$, $43 \%)(\mathrm{p} \leq 0.001)$ with an additional 279 examinations ( $34 \%$ increase). This also led to an additional 36 (51\% increase) new smear positive diagnosed without the loss in the quality of smear examinations and no difference in smear positivity rate between two periods $(\mathrm{p}=0.77)$. Similarly higher proportions of TB patients were initiated on treatment-94\% in 3Q2014 versus 91\% in 2Q2014.

\section{Discussion}

Uttarakhand is among the highest TB burden states in India (95 new smear positives/100,000 populations) [2]. Nearly $40 \%$ of the public health facilities where MCs are located (for sputum diagnosis) is categorised as "difficult to reach" [13].

Table 1. Reasons for referrals not reaching MC during 2Q2014.

\begin{tabular}{cccc}
\hline Reasons & N & $\%$ & Strategies to address \\
\hline MC not accessible & 217 & 19 & SCT \\
High transportation cost & 85 & 7 & SCT \\
Loss of daily wages & 168 & 15 & SCT \\
Inconvenient timings of operation of MC & 33 & 3 & SCT \\
Pre-occupied with agricultural activities & 138 & 12 & SCT \\
Non availability of LT & 61 & 5 & SCT where LT is available \\
Behaviour of staff & 29 & 3 & Training on soft skill \\
Alleviation of cough & 27 & 2 & Training on correct \\
& & & identification of PTBPs \\
Out migration & 18 & 2 & Linkage of source and \\
destination of referrals
\end{tabular}

2Q2014, April-June 2014; MC, microscopy centre; LT, laboratory technicians, PTBP, presumptive TB patients; SCT, sputum collection and transportation. 
Table 2. Change in the PTBPs identified and examined by referrals and sputum collection and transportation before and after the intervention.

\begin{tabular}{ccccccccc}
\hline \multirow{2}{*}{ Variables } & \multicolumn{2}{c}{$2 \mathrm{Q} 2014$} & \multicolumn{2}{c}{$3 \mathrm{Q} 2014$} & \multicolumn{2}{c}{ Change } & p-value $^{*}$ \\
\cline { 2 - 9 } & $\mathrm{n}_{1}$ & $\%$ & $\mathrm{n}_{2}$ & $\%$ & $\mathrm{n}$ & $\%$ & \\
\hline PTBPs identified & 1907 & -- & 2321 & -- & 414 & 22 & -- \\
Referred for sputum examination & 1413 & 74 & 1466 & 63 & 53 & 4 & $<0.001$ \\
Referrals reached and examined & 261 & 18 & 241 & 16 & -20 & -8 & 0.14 \\
Smear positives diagnosed through referrals & 34 & 13 & 24 & 10 & -10 & -29 & 0.29 \\
Sputum collected and transported & 494 & 26 & 855 & 37 & 361 & 73 & $<0.001$ \\
Smear positives diagnosed through SCT & 36 & 7 & 82 & 10 & 46 & 128 & 0.06 \\
Total examined & 817 & 43 & 1096 & 47 & 279 & 34 & $<0.001$ \\
Total smear Positive diagnosed & 70 & 9 & 106 & 10 & 36 & 51 & 0.77 \\
Total initiated on treatment & 64 & 91 & 100 & 94 & 36 & 56 & 0.45 \\
\hline
\end{tabular}

2Q2014 = April-June 2014, 3Q2014 = July-September 2014, ${ }^{*}$ Chi-square test for comparison of proportion, Change; $\mathrm{n}=\mathrm{n}_{2}-\mathrm{n}_{1}, \%=\left(\mathrm{n}_{2}-\mathrm{n}_{1}\right) / \mathrm{n}_{1} \times 100$, PTBPs $=$ Presumptive TB patients, $\mathrm{SCT}=$ Sputum Collection and Transportation.

As a result one would need to travel to district hospitals and this would incur additional cost for transportation, stay at district and loss of day wages. For these reasons, the referrals of the identified PTBPs from these community will have higher LTRs. Higher LTRs are associated with distance from health facility $(>10$ $\mathrm{km}$ ) and farming; results in delay in diagnosis and treatment [14] [15]. Despite promoting SCT in 3Q2014, 63\% of PTBPs patients were still referred for diagnosis for several reasons; 1) village to MC distance was $>40 \mathrm{~km}$ and CVs were reluctant to travel twice to collect spot and early morning samples, 2) PTBPs denied for SCT, and 3) PTBPs opted for referrals. Other reasons that could have contributed in LTR are; a) PTBPs visited MC without the referral slips and had not been registered as project referrals or visited another MC of their choice, b) visited qualified and unqualified private practitioners not engaged by the project, c) might have related the symptoms with underlying chronic conditions or to smoking or to seasonal cold environment condition and did not visit the MC, d) underlying stigma with $\mathrm{TB}$, difficult terrain, limited availability of transportation facility, and need for multiple visits [15] [16].

The results of this study highlight SCT services could increase number of PTBPs examined for Tuberculosis in hard-to-reach areas. Secondly, community interventions activities like ACF need to be supported by SCT services for early identification of PTBPs and reduce LTR [17] [18]. In this study, SCT alone contributed to the diagnosis of additional $128 \%$ smear positive TB patients. The smear positivity rate for SCT was 10\% (82/855) and 7\% (32/494) in 3Q2014 and 2Q2014, respectively, highlight the quality sputum collected for examination. Additional training on quality sputum collection and hands-on guidance note to CVs in 3Q2014 could have improved the quality of SCT and overall increase in smear positivity. The project focused on the smear positive cases and those PTBPs who were negative on smear examinations were referred for the chest 
$\mathrm{X}$-rays, and these were not recorded under project or as project contribution.

Conceptually, SCT would support early diagnosis, minimize TB infection transmission in community, reduce the number of doctor visits thereby preventing delay in diagnosis and treatment, and also contribute to additional $\mathrm{TB}$ cases in the hard-to-reach communities [19] [20]. SCT services also provide patient centred approach as the communities have language barriers, are selfstigmatized and discriminated which are prevalent in marginalized and vulnerable communities. The cost of identifying one TB patient through these interventions is estimated to be less than 100 USD [21]. However, a cost utility analysis of this intervention is underway.

Few limitations to this study are, 1) findings of the study are applicable to similar hard-to-reach hilly terrains where there is difficulty in reaching the health facilities 2) routine project data reports were used and lacks information on age and gender, 3) the project focused on smear positive cases only and other TB cases, like smear negative and extra-pulmonary were not included.

\section{Conclusion}

The results of this study highlight the need for establishment of SCT services in hard-to-reach areas. Forty-six additional new sputum smear positive TB patients were identified by offering SCT services which could have been missed by referral alone. In the absence of these services, PTBPs would have sought care from various service providers and not notified to the system contributing to the missing cases. This strategy would benefit TB programmes, however, one needs to prioritize the areas and create a network of volunteers to offer quality SCT services in hard-to-reach locations in India and elsewhere.

\section{Acknowledgements}

Authors would like to thank all the State and District TB officials and laboratory technicians for supporting the examination of presumptive TB patients at the microscopy centre. In addition, we would also like to thank all Project Axshya staff implementing project in Uttarakhand.

\section{Funding}

Project Axshya is an Advocacy, Communication and Social Mobilization project implemented across 21 states (including Uttarakhand) in India is supported through The Global Fund grant.

\section{Conflict of Interest}

Authors declare there is no conflict of interest.

\section{References}

[1] World Health Organization (2015) Tuberculosis Control in South-East Asia Region. Annual Report 2015. World Health Organization, New Delhi. 
[2] Government of India (2015) TB India 2015: Revised National TB Control Programme; Annual Status Report. Ministry of Health and Family Welfare, Central TB Division, New Delhi.

[3] Sudha, G., Nirupa, C., Rajasakthivel, M., et al. (2003) Factors Influencing the Care-Seeking Behaviour of Chest Symptomatics: A Community-Based Study Involving Rural and Urban Population in Tamil Nadu, South India. Tropical Medicine and International Health, 8, 336-341. https://doi.org/10.1046/j.1365-3156.2003.01010.x

[4] Satyanarayana, S., Nair, S.A., Chadha, S.S., et al. (2011) From Where Are Tuberculosis Patients Accessing Treatment in India? Results from a Cross-Sectional Community Based Survey of 30 Districts. PLoS ONE, 6, e24160.

https://doi.org/10.1371/journal.pone.0024160

[5] Mushtaq, M., Majrooh, M., Ahmad, W., et al. (2010) Knowledge, Attitudes and Practices Regarding Tuberculosis in Two Districts of Punjab, Pakistan. International Journal of Tuberculosis and Lung Diseases, 14, 303-310.

[6] Balasubramanian, R., Garg, R., Santha, T., et al. (2004) Gender Disparities in Tuberculosis: Report from a Rural DOTS Programme in South India. International Journal of Tuberculosis and Lung Diseases, 8,323-332.

[7] World Health Organization (2013) Global Strategy and Targets for Tuberculosis Prevention, Care and Control after 2015. World Health Organization, Geneva.

[8] Government of India (2012) National Strategic Plan (2012-2017). Ministry of Health and Family Welfare, Central TB Division, New Delhi.

[9] The Union (2015) Project Axshya Activity Report 2014-15. The Union South East Asia Office, TB and Communicable Disease, New Delhi.

[10] Government of Uttarakhand (2014) Uttarakhand at a Glance (2013-2014). Government of Uttarakhand, Directorate of Economics and Statistics, Dehradun.

[11] Achyut, P., Benson, A., Calhoun, M., et al. (2016) Impact Evaluation of the Urban Health Initiative in Urban Uttar Pradesh, India. Contraception, 93, 519-525. https://doi.org/10.1016/j.contraception.2016.02.031

[12] Government of India (2005) Technical and Operational Guideline for Tuberculosis Control. Ministry of Health and Family Welfare, Central TB Division, New Delhi.

[13] Government of India (2010) Health Facilities-Inaccessibility. National Health Systems Resource Centre, New Delhi.

http://www.nhsrcindia.org/index.php?option=com_content\&view=article\&id=288 \&Itemid $=724$

[14] Tripathy, J.P., Sathyanarayana, S., Naidoo, P., et al. (2013) Is Physical Access an Impediment to Tuberculosis Diagnosis and Treatment? A Study from a Rural District in North India. Public Health Action, 3, 235-239.

https://doi.org/10.5588/pha.13.0044

[15] Saifodine, A., Gudo, P.S., Sidat, M. and Black, J. (2013) Patient and Health System Delay among Patients with Pulmonary Tuberculosis in Beira City, Mozambique. BMC Public Health, 13, 559. https://doi.org/10.1186/1471-2458-13-559

[16] Sreeramareddy, C.T., Qin, Z.Z., Satyanarayana, S., et al. (2014) Delays in Diagnosis and Treatment of Pulmonary Tuberculosis in India: A Systematic Review. International Journal of Tuberculosis and Lung Diseases, 18, 255-266.

https://doi.org/10.5588/ijtld.13.0585

[17] Prasad, B.M., Satyanarayana, S., Chadha, S.S., et al. (2016) Experience of Active Tuberculosis Case Finding 5 Million Households in India. Public Health Action, 6, 15-18. https://doi.org/10.5588/pha.15.0035 
[18] Prasad, B.M., Satyanarayana, S. and Chadha, S.S. (2016) Lessons Learnt from Active Tuberculosis Case Finding in an Urban Slum Setting of Agra City, India. Indian Journal of Tuberculosis, 63, 199-202. https://doi.org/10.1016/j.ijtb.2016.08.006

[19] Yu, P., Jian, D., Ying, Z.Z., et al. (2014) The Feasibility of Sputum Transportation System in China: Effect of Sputum Storage on the Mycobacterial Detection. Biomedical and Environmental Sciences, 27, 982-986.

[20] Lönnroth, K., Aung, T., Maung, W., et al. (2007) Social Franchising of TB Care through Private GPs in Myanmar: An Assessment of Treatment Results, Access, Equity and Financial Protection. Health Policy Planning, 22, 156-166. https://doi.org/10.1093/heapol/czm007

[21] Rout, M., Prasad, B.M., Palorkar, T., et al. (2015) Value for Money: It Cost US\$90 to Identify a TB Patient in a Community Setting, India. $46^{\text {th }}$ World Conference on Lung Health, The International Union against Tuberculosis and Lung Disease, Cape Town, 2-6 December 2015, S123. 